\title{
Site Specific Preparation of Powders for High-Resolution Analytical Electron Microscopy Using a $\mathrm{Ga}^{+}$Focused Ion Beam
}

\author{
Suzy Vitale ${ }^{1}$, Joshua D. Sugar ${ }^{1}$, Patrick J. Cappillino ${ }^{2}$, Lucille A. Giannuzzi ${ }^{3}$, and David B. Robinson ${ }^{1}$ \\ ${ }^{1}$ Sandia National Laboratories, Livermore CA, USA \\ ${ }^{2}$ University of Massachusetts Dartmouth, Chemistry and Biochemistry Department, North Dartmouth, \\ MA, USA \\ ${ }^{3}$ EXpressLO LLC, 5483 Lee St., Unit 12, Lehigh Acres, FL 33971, USA
}

Preparation of powders for high-resolution microscopy presents specific challenges not present in bulk materials. Nanoscale powders (diameters $<\sim 100 \mathrm{~nm}$ ) can be directly deposited on to a TEM grid with drop casting and are usually thin enough for electron transparency in a $200 \mathrm{kV}$ instrument or above. Microscale powders (diameters $\sim 1 \mathrm{um}$ ), on the other hand, must be thinned to electron transparency for high-resolution spatial and chemical analysis.

Whereas ultramicrotomy has been able to produce powder particle samples thin enough for highresolution analysis [1], this method does not allow for complementary surface analysis on the same powder particle because the particles are encapsulated in epoxy. Similarly, conventional TEM sample preparation cannot accommodate materials of the size and structure of powder particles without also encapsulating the particles in a binding agent $[2,3]$. Any technique that fully encapsulates powder material limits analysis of particle surfaces, and the ability for site specific TEM specimen preparation is reduced using conventional specimen preparation methods. Additionally, both of these methods can take days or weeks to prepare an electron transparent TEM sample.

Powder particles have been prepared using focused ion beam (FIB) methods before [4, 5]. Using FIB and dual platform scanning electron microscope (SEM) methods, the surface of particles may be analyzed by SEM and site-specific regions may be identified for subsequent TEM analysis. In addition, electron beam induced deposition combined with ion beam induced deposition can be used to protect the particle surface morphology during cross-sectioning. Here, we will demonstrate a technique that enables both surface microscopy and high-resolution cross-sectional analytical microscopy on the same powder particle with diameters larger than $1 \mathrm{um}$. We use an EXpressLO ${ }^{\mathrm{TM}}$ ex situ [6] lift out system to mount individual powder particles to a half-grid [4]. We use multiple strategies for viewing and obtaining energy-dispersive x-ray spectroscopy (EDS) information from samples with high topographical relief. Specific FIB milling parameters can accommodate agglomerates of materials that disintegrate and collapse rapidly under the milling action of the ion beam as shown in Figure 1, where low beam current and high beam overlap prevents particle disintegration [7]. By utilizing this FIB method, we are able to efficiently prepare TEM samples of modified powder particles for both high-resolution EDS analysis and imaging (Figure 2) in just a matter of hours [8]. 
References:

[1] M. Homer et al., Microscopy and Microanalysis 21 (Suppl 3) (2015) p. 1815.

[2] H.M. Wen et al., Microscopy and Microanalysis 21 (2015), p. 1184.

[3] M.D. Ong et al., Chemistry of Materials 24 (2012), p. 996.

[4] B.I. Prenitzer et al., Metallurgical and Materials Transactions A, 29A, (1998), p. 2399.

[5] L.A. Giannuzzi and F.A. Stevie, “Introduction to Focused Ion Beams”, Springer (2005), p. 222-223.

[6] L.A. Giannuzzi et al., Microscopy and Microanalysis 21 (2015), p. 1032.

[7] W. Brostow et al., Materials Letters 61 (2007), p. 1333.

[8] Sandia National Laboratories is a multi-program laboratory managed and operated by Sandia Corporation, a wholly owned subsidiary of Lockheed Martin Corporation, for the U.S. Department of Energy’s National Nuclear Security Administration under contract DE-AC04-94AL85000.
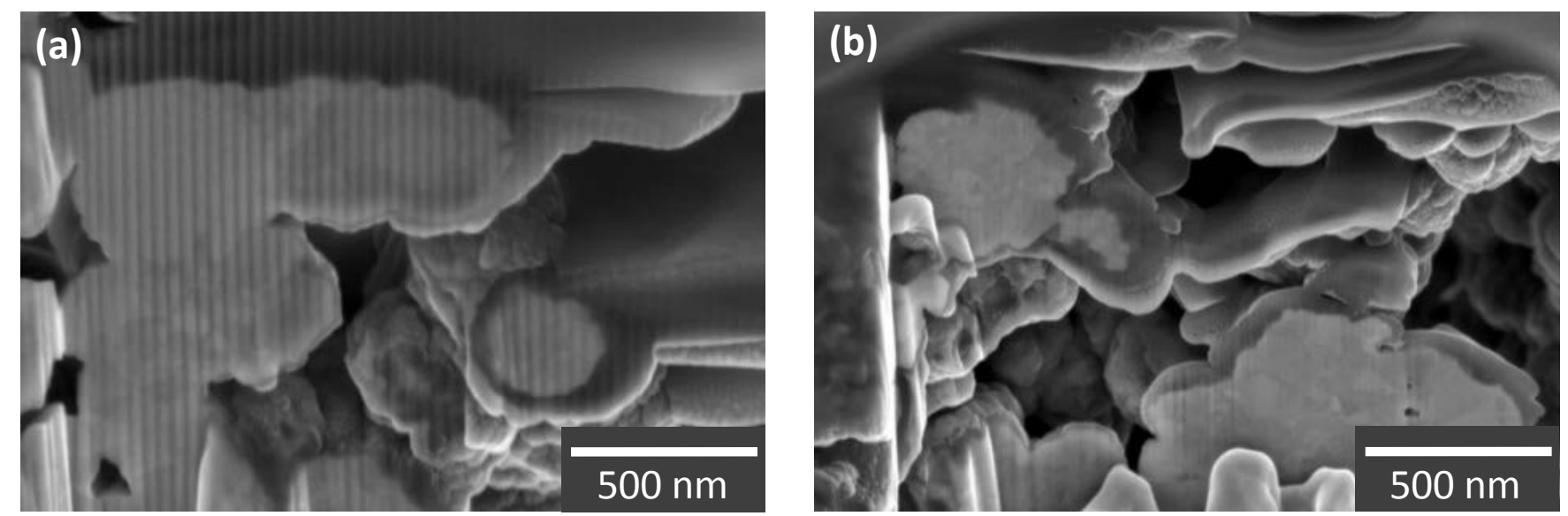

Figure 1. Development of milling strategy: (a) 80pA, -200\% overlap, (b) 80pA, -25\% overlap.
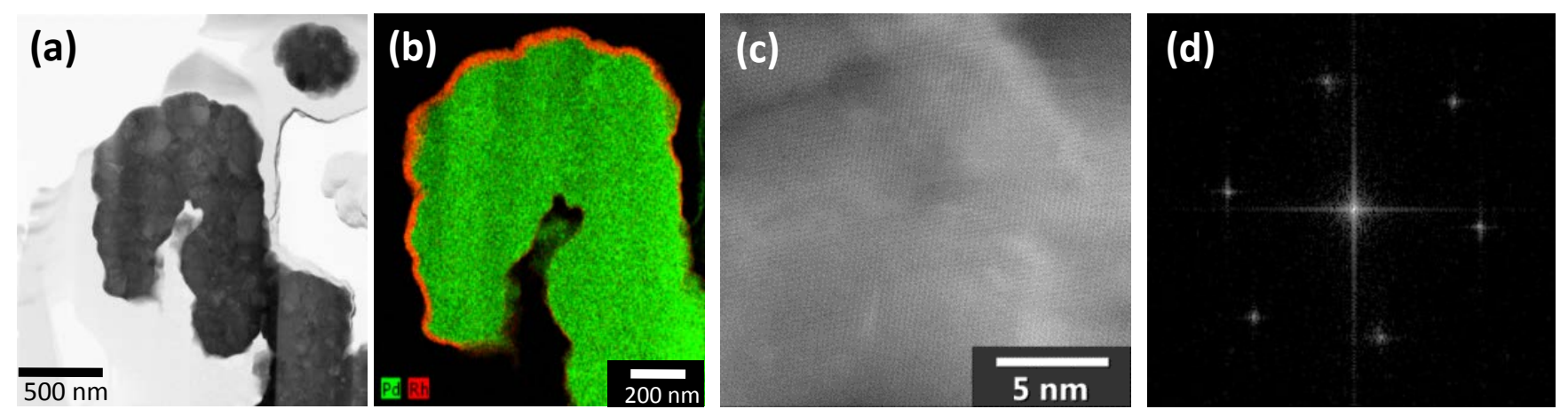

Figure 2. (a) BF STEM image of final thinned (80 nm thick), electron transparent Pd-alloy powder particle sample prepared in a $\mathrm{Ga}^{+}$FIB. EDS analysis reveals a (b) $20 \mathrm{~nm}$ Rh surface layer. (c) Atomic resolution HAADF STEM image of the same particle and (d) corresponding FFT. 